\title{
TMPRSS2 As an Influential Human Gene for COVID-19
}

\author{
Sepideh Abdollahi ${ }^{1}$ and Pantea Izadi ${ }^{1,}{ }^{*}$ \\ ${ }^{1}$ Department of Medical Genetics, School of Medicine, Tehran University of Medical Sciences, Tehran, Iran \\ "Corresponding author: Department of Medical Genetics, School of Medicine, Tehran University of Medical Sciences, Tehran, Iran. Email: p-izadi@sina.tums.ac.ir
}

Received 2021 September 07; Accepted 2021 November 03.

\begin{abstract}
In December 2019, the new virus, COVID-19, emerged and led to a pandemic respiratory acute disease. Almost all countries have experienced different rates of morbidity and mortality. These differences can be attributed to factors such as a diagnostic test capacity for COVID-19 and the health system efficiency. Besides the differences between countries related to the COVID-19 management, different patients represent a diverse range of clinical symptoms, from outpatient to patients admitted to the intensive care unit (ICU) due to the severity of symptoms. To gain deeper insights into such disparities in the severity of COVID-19 clinical presentations, epidemiological studies have reported risk factors such as old age, male sex, underlying chronic diseases such as diabetes, inflammatory and cardiovascular diseases, which have a bearing on susceptibility to COVID-19. In addition to these risk factors, the molecular mechanism involved in the virus entry process has been under investigation. Apart from a well-known protein called ACE2 (angiotensin-converting enzyme 2), which plays the receptor role for COVID-19, another essential protein in this pathway is TMPRSS2 (transmembrane protease, serine 2). This protease has a crucial role in effective membrane integration between the virus and the target cell. This process can affect the severity of the infection and the mortality rate of the disease. Thus, it seems that understanding the role of TMPRSS2 in COVID-19 infection can help better management by designing TMPRSS2 inhibitors drugs. Given the variants of the TMPRSS2 gene, which are associated with the severity of symptoms, people exposed to severe forms of this disease can be identified before the deterioration of the disease to adopt appropriate therapeutic approaches. Therefore, this study focused on the different levels of the TMPRSS2 interactions with COVID-19 virus and disease severity.
\end{abstract}

Keywords: SARS-CoV-2, COVID-19, TMPRSS2

\section{Context}

In December 2019, China and a few months later, 184 other countries faced a global pandemic caused by SARSCoV-2 (severe acute respiratory syndrome Coronavirus 2 ) or COVID-19, which is the main agent repressible for the spread of recent pneumonia $(1,2)$. COVID-19 is the seventh strain of the coronavirus and the fourth member of the betacoronaviridae family (3). Among coronavirus strains, 229E, HKU1, OC43 and NL63 have slight pathogenicity, while the SARS (severe acute respiratory syndrome) and MERS (Middle East respiratory syndrome) can lead to fatal pneumonia (4). The new strain of coronavirus, COVID-19, has a significantly higher transmission rate compared to other strains of this family(5). This substantial transmission rate has enabled the virus to spread to more than 184 countries and transforms into a pandemic as opposed to SARS and MERS, which only infected 29 and 27 countries, respectively $(6,7)$. According to the WHO (World Health Organization) as of 3 September 2021, there are 218,946,836 confirmed cases of COVID-19 disease and 4,539,723 deaths worldwide
(8).

COVID-19 can be transmitted by mucus droplets of cough, sneeze, exhalation or physical contact from one person to another $(9,10)$. The common clinical symptoms of COVID-19 include shortness of breath or difficulty breathing, fever and chills, diarrhea or vomiting, body or muscle pain, sudden loss of taste and smell and unprecedented dizziness $(11,12)$.

The notable point about the clinical symptoms is that the severity and range of clinical symptoms vary from person to person. This difference can be attributed to risk factors such as old age, heart disease, diabetes, respiratory diseases, liver or kidney disease, obesity, smoking, pregnancy, use of immunosuppressive drugs, inflammatory chronic disorder, autoimmune diseases and male gender (13-17). However, the diversity of clinical symptoms in COVID-19, as in many other multifactorial diseases, can be influenced by the genetic background of individuals. Therefore, one of the interesting topics about COVID-19 is its genetic susceptibility. One approach to track the genes and variants that affect the disease severity is to study the molecular 
pathway of the COVID-19 virus entering cells, its replication and protein-encoding genes involved in this pathway (18-21). The first step to diagnose the entry of the COVID-19 virus into the cell is to identify cell surface receptor (ACE2) and membrane integration using the viral glycoprotein $S$ (spike). As soon as the virus enters the host body, the $\mathrm{S} 1 \mathrm{sub}$ unit of the S protein binds to the RBD (receptor-binding domain) and leads to the detection of the receptor, ACE2. On the other hand, the S2 subunit of S protein is involved in membrane integration, allowing the virus to enter the target cell $(22,23)$. It should be noted that apart from ACE2, another key component use by the virus for effective membrane integration is proteases on the cell surface such as TMPRSS2 (type 2 transmembrane serine proteases) (24). Furthermore, the virus uses the TMPRSS2 for the priming of its $\mathrm{S}$ proteins. This process of preparation and active entry is crucial for the success of the virus in inducing clinical symptoms and death (25). Thus, it is important to investigate the role of TMPRSS2 as a protein in the molecular process of COVID-19. In this way, the molecular mechanisms involved in the pathogenesis of COVID-19 and possible therapeutic targets can be clarified. All of these points can contribute to the management of this pandemic disease.

\section{Results}

\subsection{TMPRSS2: From Gene to Protein and Its Function}

TMPRSS2 or serine 2 translucent protease, is an enzyme encoded by the TMPRSS2 gene in humans. Located on chromosome 21q22.3, this gene contains 15 exons. It is expressed in various body tissues, including lungs, intestines, kidneys, pancreas and prostate. Also, the protein encoded by this gene has serine protease property $(26,27)$. The protease activity of this protein is attributable to active sites called H296, D345 and S441 in the S1 peptidase domain of the extracellular region. The extracellular region of this protein also contains SRCR-2 (scavenger receptor cysteinerich domain 2) and LDLRA (LDL-receptor class A) domains in addition to the $S 1$ peptidase domain $(28,29)$. (Figure 1). Regarding the function of TMPRSS2, it is worth noting that this protease, located on the surface of the host cell, has a pivotal role in the glycoprotein process of the virus (30). Moreover, the glycoprotein process is essential for the entry of the virus into the host cell (31). Thus, TMPRSS2 can influence the penetration of the virus into cells through the glycoprotein process. For instance, it has been reported that the cells of the host's respiratory system are involved in the degradation of the influenza virus (hemagglutinin virus) by affecting the protease activity of TMPRSS2. Hemagglutinin degradation of this virus also is associated with the rate of infection caused by this virus and its tissue tropism $(32,33)$.
2.2. TMPRSS2: Molecular Mechanism Involved in the Entry of COVID-19 Into the Cells

COVID-19 penetrates cells through viral spike proteins (S proteins) by binding to its ACE2 receptors in target cells. Then $S$ proteins are activated during the proteolytic process. The $S$ proteins can be activated either through cathepsins, which leads to the virus entry in the cell through the endosomal pathway, or the protease property of TMPRSS2 protein on the surface of target cells, which leads to the entry of COVID-19 into the cell (34-36). According to a previous study, in human respiratory epithelial cells, circulating coronaviruses generally tend to enter cells via TMPRSS2 over the endosomal pathway (37). Also, TMPRSS2 has been involved in the proteolytic activity and activation of hemagglutinin influenza A virus (32), and it contributes to the spread of MERS-CoV infection by facilitating virus-cell attachment (38). Likewise, it is essential for the contraction of COVID-19 disease through the entry and membrane fusion of the COVID-19 virus by priming and activating $S$ proteins (39). Another major point concerning TMPRSS2 is the SRCR domain, which contains a protected amino acid called valine 160 . Previous studies have demonstrated the potential role of this amino acid in the binding of proteins, particularly proteins involved in the host's defense system $(28,40,41)$. These points exhibit that the role of TMPRSS2 is not limited to protease activity and it may be involved in the pathogenesis of COVID-19 virus by other mechanisms.

\subsection{TMPRSS2: Gene Polymorphisms and Their Association to COVID-19}

European populations have a higher rate of COVID-19 morbidity and mortality compared with East Asia populations. Comparison between these two populations regarding the variants and polymorphisms frequency of TMPRSS2 was carried out. The results showed the particular genetics variants of TMPRSS2 (rs463727, rs34624090, rs55964536, rs734056 rs4290734, rs34783969, rs11702475, rs35899679, rs35041537, rs2070788, rs9974589 and rs7364083), which can induce the higher expression of TMPRSS2 and are more frequent in European populations compared with East Asia (42). Moreover, another study has been reported SNPs, which can predict the expression of TMPRSS2 (43). Interestingly, the rs2070788 GG or rs383510 TT variant, which can lead to the overexpression of the TMPRSS2 in lung tissue, has been associated with increased susceptibility to influenza A (44). Maybe this association is related to the higher susceptibility of an individual to COVID-19 disease. However, the genetic variant of TMPRSS2, namely, p.V197M (p.Val197Met) (rs12329760), has a protective role in individuals against COVID-19 infection through its destructive effect on TMPRSS2 protease activity. This genetic variant is 


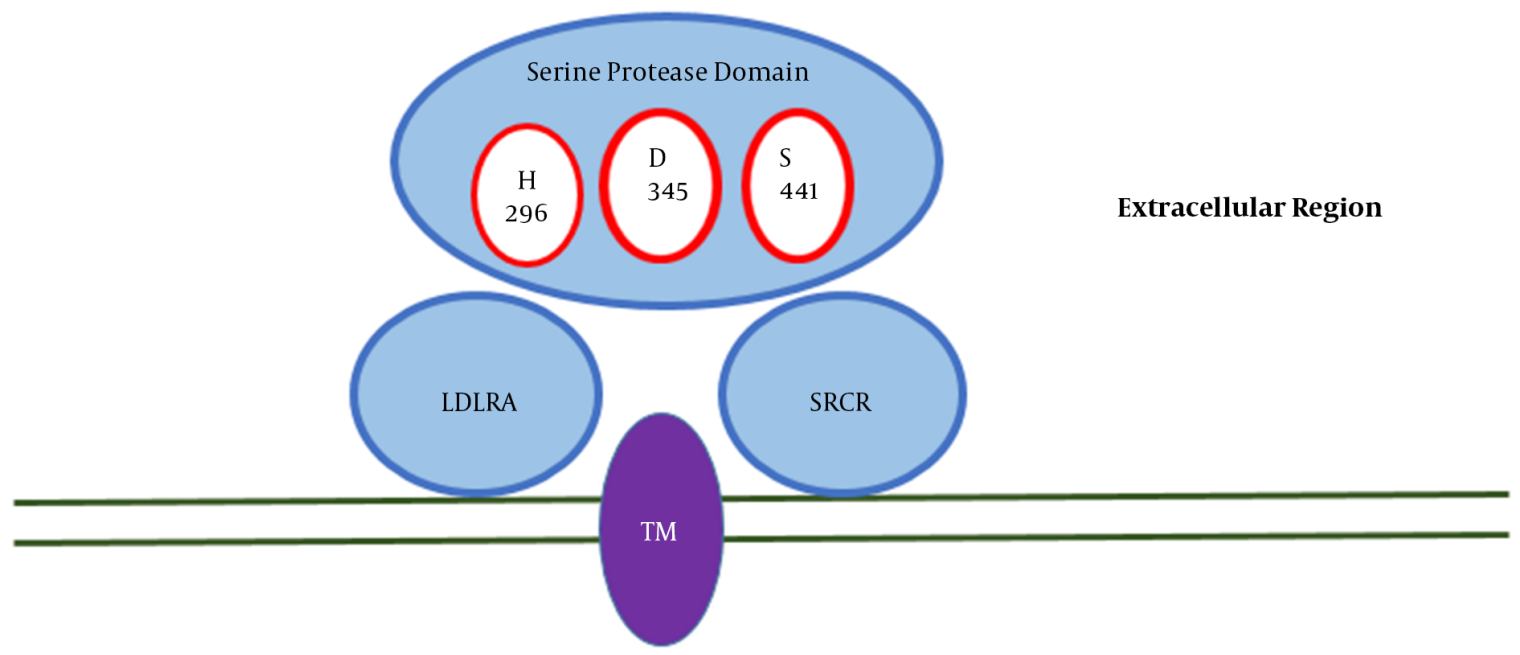

Cytoplasmic Region

Figure 1. TMPRSS2 protein domains (TM, transmembrane domain; LDLRA, LDL-receptor class A, scavenger receptor cysteine-rich domain 2).

higher in individuals who have experienced milder clinical symptoms of COVID-19 (45). Therefore, it seems that by determining the polymorphisms in the TMPRSS2 gene, it is possible to identify the individual risk of infection and susceptibility to COVID-19. In this way, we can predict the disease process at early stages to make appropriate interventions.

\subsection{TMPRSS2: Role in Gender Differences in COVID-19}

According to the available evidence, the severity of the COVID-19 disease and its mortality rate is higher in men than in women (46-49). This gender difference in the severity of COVID-19 disease may be due to various behavioral habits and the degree of social exposure leading to virus transmission differences in males and females (50). Also, this difference could be induced by the effects of sex hormones, estrogen and androgens. Molecular studies on cancer have shown that testosterone, as a major androgen, affects the production of TMPRSS2 protein in the prostate glands of men which suffer from prostate cancer. Thus, TMPRSS2 gene appears to be an androgen-responsive gene $(51,52)$. It has been suggested that a similar mechanism may occur in the lung cells of men with COVID-19. In other words, TMPRSS2 is overexpressed under the influence of testosterone in men, which facilitates the entry of COVID19 into the cell through its effect on viral S proteins. In this way, it produces various severities of the disease in men and women (53). On the other hand, in light of the importance of androgen-dependent TMPRSS2 in the entry of COVID-19 into the cell (54), studies have explored the use of
TMPRSS2 inhibitors in preventing the virus from entering the cell by inhibiting the protease activity of TMPRSS2 (39, $55)$. Informed by these important points, extensive clinical studies have been conducted to investigate the effect of using TMPRSS2 inhibitors such as camostat, nafamostat and bromhexine on the controlling of severity of COVID-19 symptoms in patients $(27,54,56)$. (Figure 2$)$.

\subsection{TMPRSS2: Role in Age Differences in COVID-19}

Evidence suggests that children and adults exhibit various degrees of clinical symptoms of COVID-19 disease (57). According to a study in China, 90\% of children are either asymptomatic or experience a mild course of disease, but elderly patients are more likely to experience severe types of the disease and be hospitalized (58). Given the importance of TMPRSS2 in the infection rate of COVID-19, many studies have looked at the association between the expression of this gene and age in the respiratory epithelial cells. The investigations have revealed a positive correlation between the expression of the TMPRSS2 gene and age. According to the results, the expression of TMPRSS2 in nasal and bronchial tissue of children is significantly lower than that of adults. (59, 60). Considering that the entrance of COVID19 into the cell depends on the expression of TMPRSS2 (39), it can be speculated that the mild clinical symptoms of COVID-19 in children could be affected by the lower levels of TMPRSS2 expression on the cell surface. 


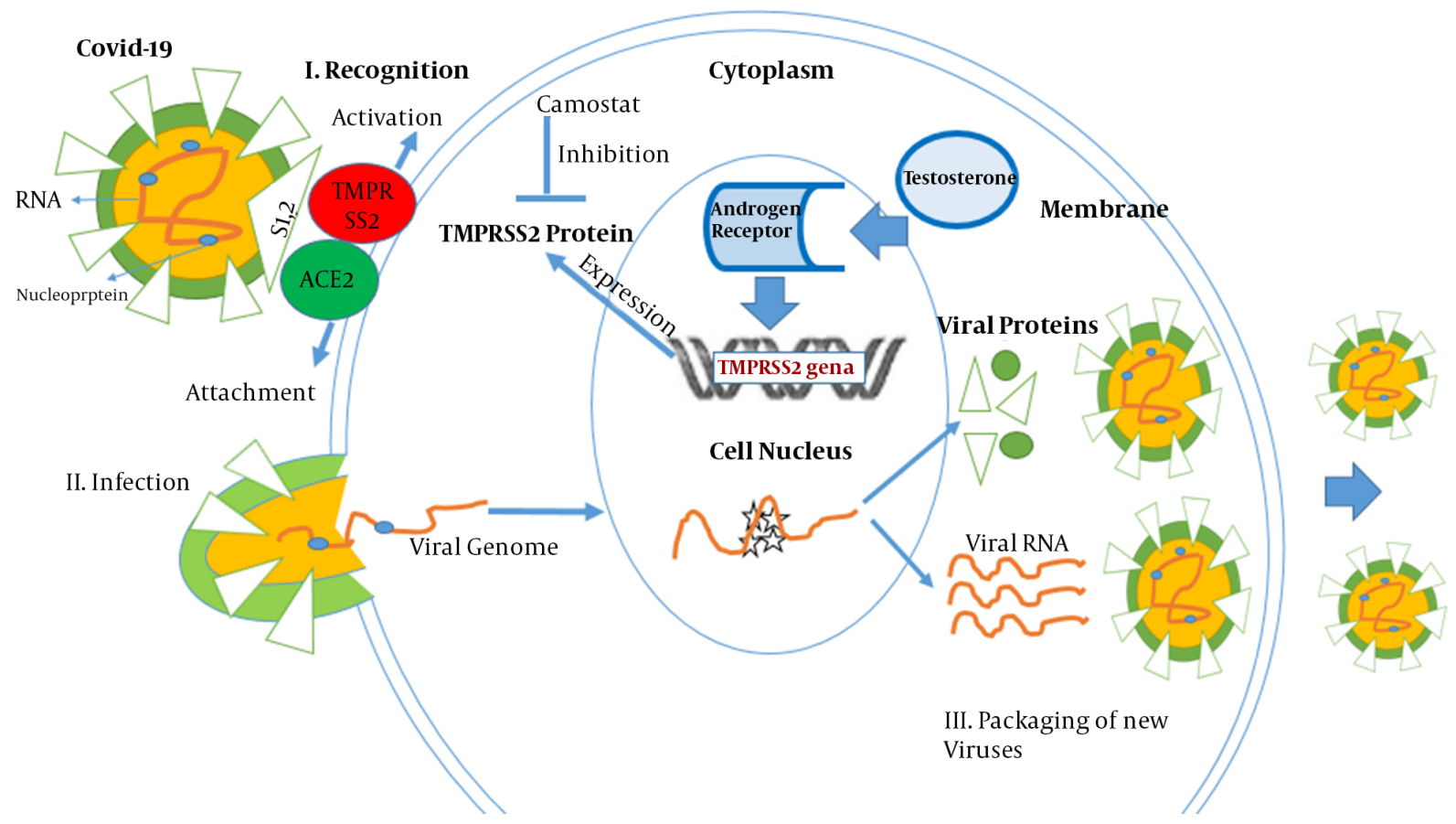

Figure 2. TMPRSS2 role in the process of COVID-19 entry to the target cell and contribution the androgens. Testosterone increases androgen receptor activity. Increased androgen receptor activity also increases transcription of the TMPRSS2 gene. COVID-19 uses ACE2 as a receptor to enter the cell and TMPRSS2 to prepare and activate its S proteins. Serine protease inhibitors such as camostat can partially block the entry of COVID-19 into cells by inhibiting TMPRSS2. (S1,2: spike glycoproteins).

\section{Conclusions}

Today, the global pandemic of COVID-19 is affecting a large number of people in different countries across the world. Investigations regarding the molecular processes involved in COVID-19 entry into the cell and proliferation of the virus are crucial for better management of the disease. In this context, the critical interaction of TMPRSS2 with ACE2 as COVID-19 receptor has been highlighted, as it can determine the severity of consequences caused by COVID-19. Therefore, further studies on different and new aspects of the TMPRSS2 gene function and its association with the severity of COVID-19 disease can lead to the development of new drug targets for better management of this disease.

\section{Footnotes}

Authors' Contribution: P.I. and S.A., study concept and design; S.A., wrote and prepared the manuscript; P.I., critical revision of the manuscript for important intellectual content. All authors read and approved the final manuscript.

Conflict of Interests: There was no conflict of interests.
Funding/Support: There was no funding/support.

\section{References}

1. Zhu N, Zhang D, Wang W, Li X, Yang B, Song J, et al. A Novel Coronavirus from Patients with Pneumonia in China, 2019. N Engl J Med. 2020;382(8):727-33. doi: 10.1056/NEJMoa2001017. [PubMed: 31978945]. [PubMed Central: PMC7092803].

2. Ciotti M, Ciccozzi M, Terrinoni A, Jiang WC, Wang CB, Bernardini S. The COVID-19 pandemic. Crit Rev Clin Lab Sci. 2020;57(6):365-88. doi: 10.1080/10408363.2020.1783198. [PubMed: 32645276].

3. Wang M, Zhang N. Focus on the 2019 novel coronavirus (SARS-CoV2). Future Microbiol. 2020;15(10):905-18. doi: 10.2217/fmb-2020-0063. [PubMed: 32524843]. [PubMed Central: PMC7291595].

4. Reina J, Lopez-Causape C, Rojo-Molinero E, Rubio R. Clinicoepidemiological characteristics of acute respiratory infections caused by coronavirus OC43, NL63 and 229E. Rev Clin Esp (Barc). 2014;214(9):499-504. doi: 10.1016/j.rce.2014.05.020. [PubMed: 24958321]. [PubMed Central: PMC7103947].

5. Gaunt ER, Hardie A, Claas EC, Simmonds P, Templeton KE. Epidemiology and clinical presentations of the four human coronaviruses 229E, HKU1, NL63, and OC43 detected over 3 years using a novel multiplex real-time PCR method. J Clin Microbiol. 2010;48(8):29407. doi: 10.1128/JCM.00636-10. [PubMed: 20554810]. [PubMed Central: PMC2916580].

6. Sutton TC, Subbarao K. Development of animal models against emerging coronaviruses: From SARS to MERS coronavirus. Virology. 2015;479-480:247-58. doi: 10.1016/j.virol.2015.02.030. [PubMed: 25791336]. [PubMed Central: PMC4793273]. 
7. Huang C, Wang Y, Li X, Ren L, Zhao J, Hu Y, et al. Clinical features of patients infected with 2019 novel coronavirus in Wuhan, China. The Lancet. 2020;395(10223):497-506. doi: 10.1016/s0140-6736(20)30183-5.

8. World Health Organization. Corona virus disease (COVID-19) dashboard. World Health Organization. 2020.

9. Salian VS, Wright JA, Vedell PT, Nair S, Li C, Kandimalla M, et al. COVID-19 Transmission, Current Treatment, and Future Therapeutic Strategies. Mol Pharm. 2021;18(3):754-71. doi: 10.1021/acs.molpharmaceut.0c00608. [PubMed: 33464914]. [PubMed Central: PMC7839412]

10. Morawska L, Milton DK. It Is Time to Address Airborne Transmission of Coronavirus Disease 2019 (COVID-19). Clin Infect Dis. 2020;71(9):23113. doi: 10.1093/cid/ciaa939. [PubMed: 32628269]. [PubMed Central PMC7454469].

11. da Rosa Mesquita R, Francelino Silva Junior LC, Santos Santana FM, Farias de Oliveira T, Campos Alcantara R, Monteiro Arnozo G, et al. Clinical manifestations of COVID-19 in the general population: systematic review. Wien Klin Wochenschr. 2021;133(7-8):377-82. doi: 10.1007/s00508-020-01760-4. [PubMed: 33242148]. [PubMed Central: PMC7689634]

12. Alimohamadi Y, Sepandi M, Taghdir M, Hosamirudsari H. Determine the most common clinical symptoms in COVID-19 patients: a systematic review and meta-analysis. J Prev Med Hyg. 2020;61(3):304-12.

13. Wolff D, Nee S, Hickey NS, Marschollek M. Risk factors for Covid19 severity and fatality: a structured literature review. Infection. 2021;49(1):15-28. doi:10.1007/s15010-020-01509-1. [PubMed: 32860214]. [PubMed Central: PMC7453858]

14. Zhang JJ, Dong X, Cao YY, Yuan YD, Yang YB, Yan YQ, et al. Clinical characteristics of 140 patients infected with SARS-CoV-2 in Wuhan, China. Allergy. 2020;75(7):1730-41. doi: 10.1111/all.14238. [PubMed: 32077115].

15. Cao W, Shi L, Chen L, Xu X, Wu Z. Clinical features and laboratory inspection of novel coronavirus pneumonia (COVID-19) in Xiangyang Hubei. MedRxiv. 2020. doi: 10.21203/rs.3.rs-15925/v1.

16. Cao M, Zhang D, Wang Y, Lu Y, Zhu X, Li Y, et al. Clinical features of patients infected with the 2019 novel coronavirus (COVID-19) in Shang hai, China. MedRxiv. 2020. doi:10.1101/2020.03.04.20030395.

17. Jin JM, Bai P, He W, Wu F, Liu XF, Han DM, et al. Gender Differences in Patients With COVID-19: Focus on Severity and Mortality. Front Public Health. 2020;8:152. doi: 10.3389/fpubh.2020.00152. [PubMed: 32411652]. [PubMed Central: PMC7201103].

18. Mohammadpour S, Torshizi Esfahani A, Halaji M, Lak M, Ranjbar R. An updated review of the association of host genetic factors with suscep tibility and resistance to COVID-19. J Cell Physiol. 2021;236(1):49-54.

19. von der Thusen J, van der Eerden M. Histopathology and genetic susceptibility in COVID-19 pneumonia. Eur J Clin Invest. 2020;50(7). e13259. doi: 10.1111/eci.13259. [PubMed: 32353898]. [PubMed Central: PMC7267318].

20. Fricke-Galindo I, Falfan-Valencia R. Genetics Insight for COVID-19 Susceptibility and Severity: A Review. Front Immunol. 2021;12:1057. doi: 10.3389/fimmu.2021.622176. [PubMed: 33868239]. [PubMed Central: PMC8047200].

21. Choudhary S, Sreenivasulu K, Mitra P, Misra S, Sharma P. Role of Genetic Variants and Gene Expression in the Susceptibility and Severity of COVID-19. Ann Lab Med. 2021;41(2):129-38. doi: 10.3343/alm.2021.41.2.129. [PubMed: 33063674]. [PubMed Central: PMC7591285].

22. Dordizadeh E, Entezari M; Saeidi; Kalantari. ACE2: Entry receptor for SARS-CoV-2. Science. 2020;367(6485):1444-8.

23. Rivellese F, Prediletto E. ACE2 at the centre of COVID-19 from paucisymptomatic infections to severe pneumonia. Autoimmun Rev 2020;19(6):102536. doi: 10.1016/j.autrev.2020.102536. [PubMed 32251718]. [PubMed Central: PMC7195011].

24. Thunders M, Delahunt B. Gene of the month: TMPRSS2 (transmembrane serine protease 2). I Clin Pathol. 2020;73(12):773-6. doi: 10.1136/jclinpath-2020-206987. [PubMed: 32873700]. [PubMed Central: PMC7470178]
25. Shang J, Wan Y, Luo C, Ye G, Geng Q, Auerbach A, et al. Cell entry mechanisms of SARS-CoV-2. Proc Natl Acad Sci U S A. 2020;117(21):11727-34. doi: 10.1073/pnas.2003138117. [PubMed: 32376634]. [PubMed Central: PMC7260975].

26. Paoloni-Giacobino A, Chen H, Peitsch MC, Rossier C, Antonarakis SE. Cloning of the TMPRSS2 gene, which encodes a novel serine protease with transmembrane, LDLRA, and SRCR domains and maps to 21q22.3. Genomics. 1997;44(3):309-20. doi: 10.1006/geno.1997.4845. [PubMed: 9325052].

27. Mollica V, Rizzo A, Massari F. The pivotal role of TMPRSS2 in coronavirus disease 2019 and prostate cancer. Future Oncol. 2020;16(27):2029-33. doi: 10.2217/fon-2020-0571. [PubMed: 32658591]. [PubMed Central: PMC7359420].

28. David A, Khanna T, Beykou M, Hanna G, Sternberg MJ. Structure, function and variants analysis of the androgen-regulated TMPRSS2, a drug target candidate for COVID-19 infection. BioRxiv. 2020. doi: 10.1101/2020.05.26.116608.

29. Afar DE, Vivanco I, Hubert RS, Kuo J, Chen E, Saffran DC, et al. Catalytic cleavage of the androgen-regulated TMPRSS2 protease results in its secretion by prostate and prostate cancer epithelia. Cancer Res. 2001;61(4):1686-92.

30. Bertram S, Heurich A, Lavender H, Gierer S, Danisch S, Perin $\mathrm{P}$, et al. Influenza and SARS-coronavirus activating proteases TMPRSS2 and HAT are expressed at multiple sites in human respiratory and gastrointestinal tracts. PLoS One. 2012;7(4). e35876. doi: 10.1371/journal.pone.0035876. [PubMed: 22558251]. [PubMed Central: PMC3340400].

31. Choppin PW, Scheid A. The role of viral glycoproteins in adsorption, penetration, and pathogenicity of viruses. Rev Infect Dis. 1980;2(1):4061. doi: 10.1093/clinids/2.1.40. [PubMed: 6994202]

32. Bottcher E, Matrosovich T, Beyerle M, Klenk HD, Garten W, Matrosovich M. Proteolytic activation of influenza viruses by serine proteases TMPRSS2 and HAT from human airway epithelium. J Virol. 2006;80(19):9896-8. doi: 10.1128/JVI.01118-06. [PubMed: 16973594]. [PubMed Central: PMC1617224].

33. Takahashi M, Sano T, Yamaoka K, Kamimura T, Umemoto N, Nishitani $\mathrm{H}$, et al. Localization of human airway trypsin-like protease in the airway: an immunohistochemical study. Histochem Cell Biol. 2001;115(3):181-7. doi: 10.1007/s004180000243. [PubMed: 11326745].

34. Gierer S, Bertram S, Kaup F, Wrensch F, Heurich A, Kramer-Kuhl A, et al. The spike protein of the emerging betacoronavirus EMC uses a novel coronavirus receptor for entry, can be activated by TMPRSS2, and is targeted by neutralizing antibodies. J Virol. 2013;87(10):550211. doi: 10.1128/JVI.00128-13. [PubMed: 23468491]. [PubMed Central: PMC3648152]

35. Belouzard S, Millet JK, Licitra BN, Whittaker GR. Mechanisms of coronavirus cell entry mediated by the viral spike protein. Viruses. 2012;4(6):1011-33. doi: 10.3390/v4061011. [PubMed: 22816037]. [PubMed Central: PMC3397359].

36. Heald-Sargent T, Gallagher T. Ready, set, fuse! The coronavirus spike protein and acquisition of fusion competence. Viruses. 2012;4(4):55780. doi: 10.3390/v4040557. [PubMed: 22590686]. [PubMed Central: PMC3347323].

37. Shirato K, Kawase M, Matsuyama S. Wild-type human coronaviruses prefer cell-surface TMPRSS2 to endosomal cathepsins for cell entry. Virology. 2018;517:9-15. doi: 10.1016/j.virol.2017.11.012. [PubMed: 29217279]. [PubMed Central: PMC7112029].

38. Du L, Yang Y, Zhou Y, Lu L, Li F, Jiang S. MERS-CoV spike protein: a key target for antivirals. Expert Opin Ther Targets. 2017;21(2):131-43. doi: 10.1080/14728222.2017.1271415. [PubMed: 27936982]. [PubMed Central: PMC5457961]

39. Hoffmann M, Kleine-Weber H, Schroeder S, Kruger N, Herrler T, Erichsen S, et al. SARS-CoV-2 Cell Entry Depends on ACE2 and TMPRSS2 and Is Blocked by a Clinically Proven Protease Inhibitor. Cell. 2020;181(2):271280 e8. doi: 10.1016/j.cell.2020.02.052. [PubMed: 32142651]. [PubMed 
Central: PMC7102627].

40. Aruffo A, Bowen MA, Patel DD, Haynes BF, Starling GC, Gebe JA, et al. CD6-ligand interactions: a paradigm for SRCR domain function? Immunol Today. 1997;18(10):498-504. doi:10.1016/s0167-5699(97)01130-4.

41. Resnick D, Pearson A, Krieger M. The SRCR superfamily: a family reminiscent of the Ig superfamily. Trends Biochem Sci. 1994;19(1):5-8. doi: 10.1016/0968-0004(94)90165-1.

42. Asselta R, Paraboschi EM, Mantovani A, Duga S. ACE2 and TMPRSS2 variants and expression as candidates to sex and country differences in COVID-19 severity in Italy. Aging (Albany NY). 2020;12(11):10087-98. doi: 10.18632/aging.103415. [PubMed: 32501810]. [PubMed Central: PMC7346072].

43. Piva F, Sabanovic B, Cecati M, Giulietti M. Expression and coexpression analyses of TMPRSS2, a key element in COVID-19. Eur J Clin Microbiol Infect Dis. 2021;40(2):451-5. doi: 10.1007/s10096-020-04089-y. [PubMed: 33245471]. [PubMed Central: PMC7693853].

44. Cheng Z, Zhou J, To KK, Chu H, Li C, Wang D, et al. Identification of TMPRSS2 as a Susceptibility Gene for Severe 2009 Pandemic A(H1N1) Influenza and A(H7N9) Influenza. J Infect Dis. 2015;212(8):121421. doi: 10.1093/infdis/jiv246. [PubMed: 25904605]. [PubMed Central: PMC7107393].

45. Monticelli M, Hay Mele B, Benetti E, Fallerini C, Baldassarri M, Furini S, et al. Protective Role of a TMPRSS2 Variant on Severe COVID19 Outcome in Young Males and Elderly Women. Genes (Basel). 2021;12(4):596. doi: 10.3390/genes12040596. [PubMed: 33921689]. [PubMed Central: PMC8073081].

46. Richardson S, Hirsch JS, Narasimhan M, Crawford JM, McGinn T, Davidson KW, et al. Presenting Characteristics, Comorbidities, and Outcomes Among 5700 Patients Hospitalized With COVID19 in the New York City Area. JAMA. 2020;323(20):2052-9. doi: 10.1001/jama.2020.6775. [PubMed: 32320003]. [PubMed Central: PMC7177629].

47. Guan WJ, Ni ZY, Hu Y, Liang WH, Ou CQ, He JX, et al. Clinical Characteristics of Coronavirus Disease 2019 in China. N Engl J Med. 2020;382(18):1708-20. doi: 10.1056/NEJMoa2002032. [PubMed: 32109013]. [PubMed Central: PMC7092819].

48. Stall NM, Wu W, Lapointe-Shaw L, Fisman DN, Giannakeas V, Hillmer MP, et al. Sex- and Age-Specific Differences in COVID-19 Testing, Cases, and Outcomes: A Population-Wide Study in Ontario, Canada. J Am Geriatr Soc. 2020;68(10):2188-91. doi: 10.1111/jgs.16761. [PubMed: 32743827].

49. Lochlainn MN, Lee KA, Sudre CH, Varsavsky T, Cardoso M, Menni C, et al. Key predictors of attending hospital with COVID19: an association study from the COVID symptom Tracker APP in 2,618,948 individuals. medRxiv. 2020. doi: 10.1101/2020.04.25.20079251.

50. Wenham C, Smith J, Morgan R. COVID-19: the gendered impacts of the outbreak. The Lancet. 2020;395(10227):846-8. doi: 10.1016/s01406736(20)30526-2.

51. Wang L, Williamson SR, Zhang S, Huang J, Montironi R, Davison $\mathrm{DD}$, et al. Increased androgen receptor gene copy number is associated with TMPRSS2-ERG rearrangement in prostatic small cell carcinoma. Mol Carcinog. 2015;54(9):900-7. doi: 10.1002/mc.22162. [PubMed: 24777847].

52. Yoo S, Pettersson A, Jordahl KM, Lis RT, Lindstrom S, Meisner A et al. Androgen receptor CAG repeat polymorphism and risk of TMPRSS2:ERG-positive prostate cancer. Cancer Epidemiol Biomarkers Prev. 2014;23(10):2027-31. doi: 10.1158/1055-9965.EPI-14-0020. [PubMed: 24925673]. [PubMed Central: PMC4184923].

53. Wadman M. Sex hormones signal why virus hits men harder. Science. 2020;368(6495):1038-9. doi: 10.1126/science.368.6495.1038. [PubMed: 32499416].

54. Cattrini C, Bersanelli M, Latocca MM, Conte B, Vallome G, Boccardo F Sex Hormones and Hormone Therapy during COVID-19 Pandemic: Implications for Patients with Cancer. Cancers (Basel). 2020;12(8):2325 doi:10.3390/cancers12082325. [PubMed: 32824674]. [PubMed Central: PMC7464909].

55. Zhou Y, Vedantham P, Lu K, Agudelo J, Carrion RJ, Nunneley JW, et al. Protease inhibitors targeting coronavirus and filovirus entry. Antiviral Res. 2015;116:76-84. doi:10.1016/j.antiviral.2015.01.011. [PubMed: 25666761]. [PubMed Central: PMC4774534].

56. Stopsack KH, Mucci LA, Antonarakis ES, Nelson PS, Kantoff PW. TMPRSS2 and COVID-19: Serendipity or Opportunity for Intervention? Cancer Discov. 2020;10(6):779-82. doi: 10.1158/2159-8290.CD-20-0451. [PubMed: 32276929]. [PubMed Central: PMC7437472]

57. Dong Y, Mo X, Hu Y, Qi X, Jiang F, Jiang Z, et al. Epidemiology of COVID-19 Among Children in China. Pediatrics. 2020;145(6). doi 10.1542/peds.2020-0702. [PubMed: 32179660].

58. Dhochak N, Singhal T, Kabra SK, Lodha R. Pathophysiology of COVID-19: Why Children Fare Better than Adults? Indian J Pediatr. 2020;87(7):537-46. doi: 10.1007/s12098-020-03322-y. [PubMed: 32410003]. [PubMed Central: PMC7221011].

59. Schuler BA, Habermann AC, Plosa EJ, Taylor CJ, Jetter C, Negretti $\mathrm{NM}$, et al. Age-determined expression of priming protease TMPRSS2 and localization of SARS-CoV-2 in lung epithelium. J Clin Invest. 2021;131(1). doi: 10.1172/JCl140766. [PubMed: 33180746]. [PubMed Central: PMC7773394].

60. Saheb Sharif-Askari N, Saheb Sharif-Askari F, Alabed M, Temsah MH, Al Heialy S, Hamid Q, et al. Airways Expression of SARS-CoV-2 Receptor, ACE2, and TMPRSS2 Is Lower in Children Than Adults and Increases with Smoking and COPD. Mol Ther Methods Clin Dev. 2020;11(18):1-6. doi: 10.1016/j.omtm.2020.05.013. [PubMed: 32537478]. [PubMed Central: PMC7242205]. 